\title{
Universiteit
}

Leiden

The Netherlands

\section{Langage, mythe et philologie dans la Philosophie des Formes} Symboliques d'Ernst Cassirer

Iribarren Baralt, L.

\section{Citation}

Iribarren Baralt, L. (2012). Langage, mythe et philologie dans la Philosophie des

Formes Symboliques d'Ernst Cassirer. Revue Germanique Internationale, 15, 95-114.

Retrieved from https://hdl.handle.net/1887/36574

Version: $\quad$ Not Applicable (or Unknown)

License: $\quad$ Leiden University Non-exclusive license

Downloaded from: $\quad$ https://hdl.handle.net/1887/36574

Note: To cite this publication please use the final published version (if applicable). 
REVUE GERMANIQUE

$15 / 2012$

INTERNATIONALE

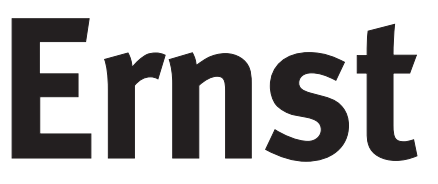

CASSIRER 


\section{Sommaire}

5 Carole Maigné, Enno Rudolph, Présentation

9 Kurt Flasch, Ernst Cassirer, interprète de Nicolas de Cues

21 Christian BERNER, Histoire et interprétation : le principe «renaissance »

37 Guido KREIs, Le problème de l'bistoricité dans une philosophie des formes symboliques

53 Enno Rudolph, Ernst Cassirer : Entre philosophie de l'histoire et historicisme

63 Carole Maigné, Herbart paradoxal ? La lecture de Cassirer

77 Marc De LaunaY, Histoire et dialectique d'une forme symbolique. La réception par Cassirer des Noms divins de Hermann Usener

95 Leopoldo Iribarren, Langage, mythe et philologie dans la Philosophie des formes symboliques d'Ernst Cassirer

115 Denis Thouard, Objectivation ou aliénation. Retour sur Cassirer, Simmel et la «tragédie de la culture »

129 Dominic KAEgi, L'éthique non écrite de Cassirer

139 Massimo Ferrari, Cassirer, Kant et l'Aufklärung

161 John KroIs, Symbolisme et phénomène de base (Basisphänomene) 


\title{
Langage, mythe et philologie dans la Philosophie des formes symboliques d'Ernst Cassirer.
}

\author{
Leopoldo Iribarren
}

À Pierre Judet de La Combe

L'une des critiques récurrentes dont fait l'objet la Philosophie des formes symboliques (1923-1929) d'Ernst Cassirer touche à une difficulté principielle du système qui consiste, comme le résume Hans Blumenberg, en un désaccord entre, d'une part, la validité propre que l'auteur attribue à chacune des parties qui le composent - mythe, art, langage et science - et, de l'autre, l'intentionnalité qui parcourt l'ensemble du système qui vise la connaissance de type scientifique et sa validité indépassable $^{1}$. Selon la première de ces deux thèses, les formes symboliques correspondent aux différentes sphères d'objectivation de l'esprit, qui seraient toutes également originaires et incommensurables. Pour illustrer son propos, Cassirer a souvent recours à l'exemple de l'image d'une ligne que l'on peut appréhender de manière différente selon l'orientation symbolique dont on l'investit. On peut en effet attribuer à la perception d'une simple ligne une signification esthétique, mais on peut aussi voir là un ornement portant une signification mythico-religieuse, ou, si l'on saisit la ligne

* Une première version de ce texte fut présentée lors du colloque international « Ernst Cassirer : Kultur und Geschichte », organisé par Carole Maigné et Enno Rudolph à Paris, les 11-12 juin 2010. Qu'ils soient ici remerciés de leur accueil, ainsi que Marc de Launay et André Laks pour les remarques très utiles qu'ils m'ont faites. Ce travail fait partie des recherches que j'ai pu mener dans le cadre du projet ANR «Présocratiques Grecs/Présocratiques Latins » (ANR-08-BLAN-0063), sous la direction d'André Laks. Les références bibliographiques de Cassirer renvoient aux Gesammelte Werke (ECW) et aux Nachgelassene Manuskripte und Texte (ECN), Felix Meiner Verlag, Hambourg, 1995-2009. Je cite les traductions françaises correspondantes quand elles existent, au cas contraire, c'est moi qui traduis.

1. Hans Blumenberg, «Hommage à Ernst Cassirer », discours de réception du prix Kuno Fischer, juin 1974, in L'imitation de la nature et autres essais esthétiques, trad. fr. I. Kalinowski et M. de Launay, Hermann Ed., Paris, 2010, (p. 159-170), p. 164. 
dans une perspective mathématique, on peut y découvrir l'image d'une fonction² Chaque monde symbolique est fondé sur un mode spécifique d'appréhension qui conditionne les catégories de temps, d'espace, de substance et de causalité. Comme l'affirme l'auteur dans l'introduction à la PFS :

Aucune de ces formations ne peut se déduire des autres ni ne peut s'y ramener purement et simplement, mais chacune d'entre elles se réfère à un mode d'appréhension de l'esprit bien défini et chacune constitue, à l'intérieur de ce point de vue et à travers lui, un aspect particulier du « réel » ${ }^{3}$.

Cette vision perspectiviste, qui met l'accent sur les caractéristiques distinctes et irréductibles des diverses activités symboliques, contraste avec cette autre vision, dépendante d'un principe dynamique d'inspiration idéaliste, selon laquelle des fonctions ou des dimensions (les deux termes sont ici interchangeables) symboliques se succèdent dans la direction d'une émancipation du sensible inscrite dans le développement culturel de l'humanité. La mobilisation dans ce contexte de la notion de fonction, que Cassirer avait déjà opposée à celle de substance dans sa doctrine du concept ${ }^{4}$, répond à l'impératif kantien de fonder l'unité du système non pas sur l'objet de connaissance, mais sur le sujet'. C'est donc une fonction de l'esprit qui à chaque fois prend en charge l'élaboration signifiante d'un contenu. Cassirer envisage, de ce point de vue, une montée progressive de la réflexivité en trois étapes qui mettent en rapport de façon idéelle une fonction et une forme symbolique. Il y a d'abord la fonction de l'expression (Ausdruck) qui transforme la force des impressions sensibles dans des éléments signifiants, tels les images mythiques qui stabilisent les réponses affectives. On passe ensuite à la fonction de présentation (Darstellung) propre aux propositions du langage courant, lequel, au moyen de concepts, cherche à restituer un état objectif des choses et vise l'intersubjectivité. Enfin, on arrive à la pure signification (reine Bedeutung) à l'œuvre dans les systèmes axiomatiques, qui se détachent définitivement des schèmes intuitifs de la présentation pour accéder à un monde symbolique

2. Ernst Cassirer, Philosophie der symbolischen Formen I. Die Sprache (1923), ECW 11, p. 28 [trad. fr. O. Hansen-Love et J. Lacoste, Minuit, Paris, 1972, p. 38-39]. Voir aussi « Das Symbolproblem und seine Stellung im System der Philosophie » (1927), ECW 17, (p. 253-282), p. 257 [trad. française d'E. Dufour, « Le système symbolique et sa place dans la philosophie », in : Cohen, Natorp, Cassirer (et alii), Néokantismes et théorie de la connaissance, Paris, Vrin, 2000, (p. 203-222), p. 207].

3. Ibid. p. 7 [trad. fr. modifiée, p. 19]

4. Cf. Enst Cassirer, Substanzbegriff und Funktionsbegriff. Untersuchungen über die Grunfragen der Erkenntniskritik, (1910), ECW 6 [trad. fr. de P. Caussat, Substance et fonction. Éléments pour une théorie du concept, Paris, Minuit, 1977].

5. Cf. Ernst Cassirer, « Der Begriff der symbolischen Form im Afbau der Geisteswissenschaften » (1921-1922), ECW 16, p. 75-104 [trad. fr. de J. Carro, «Le concept de forme symbolique dans l'édification des sciences de l'esprit », in Cassirer, Trois essais sur le symbolique, Paris, Ed. du Cerf, p. 9-37]. 
gouverné par des principes généraux d'agencement ${ }^{6}$. Quant à l'art, qui occupe une position complexe dans ce schéma, il se situerait en tension entre le monde de l'expression pure et celui de la présentation pure ${ }^{7}$. Selon cette approche, qui ne cache pas sa dette vis-à-vis de la phénoménologie hégélienne, le symbole constitue l'instrument au moyen duquel la conscience contient potentiellement le tout, dégageant à chaque fois des contenus de plus en plus libres de l'emprise sensible.

Les deux thèses que l'on vient de résumer coexistent non seulement au sein de l'œuvre majeure qu'est la PFS, mais aussi dans un certain nombre de textes des années 1920 qui concernent son fondement méthodologique. Elles sont, en quelque sorte, l'expression de deux directions théoriques virtuellement contradictoires entre elles qui traversent la pensée cassirérienne dans la période dite de Hambourg. On trouve là, d'une part, un élargissement important de la théorie de la connaissance - qui dans l'approche néokantienne de l'école de Marbourg concernait prioritairement les sciences mathématiques et physique - vers la totalité des sphères de l'activité humaine. De ce fait, la théorie de la connaissance, qui fut le premier grand thème philosophique de Cassirer, se trouve refondue en une théorie générale de la culture, où le terme de culture ne désigne pas une totalité donnée, mais l'ensemble de relations entre des orientations symboliques diverses et parfois opposées où l'esprit est simultanément objectivé. Mais, d'autre part, Cassirer ne cherche pas moins, dans la perspective de ce qu'il appelle une phénoménologie de la culture, à élucider la logique qui préside à la différenciation des mondes symboliques et à retracer la dynamique de leur succession historique commandée par la finalité cognitive. Dans la mesure où l'accent est mis sur l'histoire progressive des représentations abstraites de la conscience, le projet de la PFS se rapproche du modèle historiographique de la Geistesgeschichte - avec cependant cette réserve : la reconnaissance, ne serait-ce que relative, par Cassirer des conditions réelles qui déterminent le sens des différents contenus de la pensée. Le conflit principiel que soulève la concomitance de ces deux orientations théoriques ne fut jamais traité de manière explicite par Cassirer. Faisant preuve de modestie critique, il se contentait d'affirmer à propos de la PFS qu'elle « n'aspirait pas à fonder et à parachever une construction, mais voulait seulement en jeter les bases $»^{8}$. Plus récemment, dans un hommage à son auteur, Habermas a analysé cette difficulté à la lumière de la position problématique que le langage occupe

6. Cette triade apparaît formulée pour la première fois dans la conférence de 1927, « Das Symbolproblem », ECW 17 (p. 253-282), soit trois ans après la parution du deuxième volume de la PSF. Elle sera systématiquement reprise et développée dans le troisième volume, Phänomenologie der Erkenntnis, publié en 1929.

7. Pour une analyse de la position que l'art occupe dans le système des formes symboliques, je renvoie à la thèse doctorale de Muriel Van Vliet, Esthétique et anthropologie selon Ernst Cassirer. Une introduction aux sciences bumaines, Université de Rennes 1, 2011.

8. Ce commentaire fait partie d'une réponse aux critiques formulées par Konrad Marc-Wogau à l'égard de la structure logique du concept. Voir Ernst Cassirer, «Zur Logik des Symbolbegriffs » (1938), ECW 22 (p. 112-139), p. 137 [trad. fr. de J. Carro, « De la logique du concept de symbole », in Cassirer, Trois essais sur le symbolique, Paris, Ed. du Cerf, 1997, p. 140]. 
dans l'architectonique du système : à la fois forme symbolique à part entière et milieu où prennent forme les principales activités de l'esprit'. Cette position conflictuelle trouve son origine, comme le suggère Habermas, dans l'usage partiel que Cassirer fait du linguistic turn humboldtien. En effet, le statut du langage comme forme symbolique repose chez Cassirer sur un développement de la ligne sémantique de la philosophie de Humboldt (bien que la ligne pragmatique ne soit pas totalement absente des analyses ${ }^{10}$ ). Mobilisant la fonction cognitive d'ouverture au monde que Humboldt attribue au langage, Cassirer déploie dans le premier volume de la PFS une analyse sémantique des structures de sens de type catégorial qui sont inhérentes à la forme linguistique en tant que telle. Dans cette perspective, la légitimité propre du langage comme forme objective de la conscience est strictement fondée sur sa faculté d'articulation catégorielle du monde. Cette vision contraignante - dans la mesure où elle surdétermine la fonction cognitive du langage au détriment d'autres fonctions qui selon Humboldt lui sont tout aussi inhérentes, comme l'expression ou la communication - vaut au langage la position subordonnée qu'il occupe dans le système de Cassirer, entre le mythe et la connaissance théorique. Or le langage n'est pas moins, dans ce même système, le milieu où prennent forme et s'objectivent d'autres directions symboliques de la conscience, comme le mythe ou la poésie, dont la fonction primordiale n'est pas de forger les pensées ni de présenter des faits ; sans oublier qu'il est aussi le milieu où sont thématisées les diverses activités de l'esprit. Pour surmonter cette difficulté architectonique, il aurait fallu, selon Habermas :

transformer la priorité heuristique dont jouissent dans ses recherches l'analyse transcendantale du langage et du monde vécu linguistiquement constitué en une priorité systématique. Il aurait fallu donner au langage et au monde vécu une position centrale dans la construction des formes symboliques. En faisant ce pas, Cassirer aurait surmonté sa vision épistémologiquement contraignante et résolu le conflit qui poursuit sa philosophie des formes symboliques entre, d'une part, le perspectivisme des mondes également originaires, et, de l'autre, le pouvoir émancipateur de la mise en forme symbolique ${ }^{11}$.

Cassirer n'aurait sans doute pas pu justifier théoriquement la centralité systématique du langage sans entrer en contradiction avec sa propre thèse sur la parité irréductible des mondes symboliques. Mais cette difficulté qui touche à la légitimité abstraite du système ne l'a pas empêché, au cours de certaines analyses, d'attribuer de fait au langage cette position centrale. Dans les pages qui suivent, je me propose de montrer qu'une relocalisation du langage dans le système s'opère notamment dans la confrontation théorique avec la forme symbolique du mythe. Le glissement vers une priorité systématique du langage est effectivement perceptible à travers les trois princi-

9. Jürgen Habermas, « Die befreiende Kraft der symbolischen Formgebung », in D. Frede et R. Schmücker (éds.), Ernst Cassirers Werk und Wirkung, Darmstadt, Wiss. Buchges., 1997, p. 79-104.

10. Voir à ce propos l'étude de Birgit Recki, Kultur als Praxis. Eine Einführung in Ernst Cassirers Philosophie der symbolischen Formen, Berlin, Akademie Verlag, 2004.

11. Ibid., p. 99-100. 
paux textes que Cassirer consacre au mythe entre 1921 et 1925, à savoir « La forme du concept dans la pensée mythique » (1921-1922), La pensée mythique (1925) qui constitue le deuxième volume de la PFS et Langage et mythe $(1925)^{12}$. S'il est vrai que ces trois textes s'inscrivent dans une même tentative globale d'explication du mythe comme dimension originaire de l'expression symbolique, leur approche diffère sensiblement en fonction de l'intérêt de la connaissance qui à chaque fois oriente la recherche. Dans le texte de 1921, sans doute le plus proche des problématiques néokantiennes, il s'agit principalement de déterminer le mode de conceptualisation à l'œuvre dans la pensée mythique en son rapport avec la forme « achevée » du concept scientifique. L'approche change dans La pensée mythique où la forme symbolique du mythe fait surtout l'objet d'une description phénoménologique (le mythe comme forme de vie). Enfin, dans Langage et mythe, Cassirer aborde la forme mythique comme phénomène fondamentalement langagier à partir d'une « théorie générale des significations $»^{13}$ largement inspirée de l'ouvrage Götternamen (1896) de Hermann Usener. La thèse que je défendrai ici est que la mobilisation du langage en direction d'une priorité systématique culmine dans Langage et mythe, où l'on voit comment Cassirer tire de la philologie spéculative pratiquée par Usener dans Götternamen le modèle d'un mouvement dialectique immanent au langage qui jouera dans son système le rôle fondamental de moteur de la différenciation des mondes symboliques.

\section{L'embarras du mythe}

Contrairement à l'analyse du langage comme forme symbolique, où la conception transcendantale de Humboldt s'imposait naturellement comme fil conducteur, la diversité des approches dont fait l'objet chez Cassirer la conscience mythique témoigne, on le verra, d'un réel embarras méthodologique.

Sa première étude consacrée à la forme de pensée mythique est une conférence prononcée en juillet 1921 à la Religionswissenschaftlichen Gesellschaft de Hambourg et présentée comme une esquisse s'inscrivant dans le vaste projet d'une philosophie des formes symboliques. Animé par Fritz Saxl, qui dirigeait alors la Kulturwissenschaftliche Bibliothek Warburg, Cassirer se décide à publier une version élargie de la conférence dans la série Studien der Bibliothek. Warburg ${ }^{14}$. C'est par ail-

12. Ernst Cassirer, « Die Begriffsform im mythischen Denken » (1922), ECW 16, p. 3-73 [trad. fr. de J. Carro, in Cassirer, Trois essais sur le symbolique, Paris, Ed. du Cerf, 1997, p. 39-111] ; Das mythische Denken (1925), ECW 12 [trad. fr. de J. Lacoste, Paris, Ed. de Minuit, 1972] ; Sprache und Mythos (1925), ECW 16, p. 227-311 [trad. fr. de O. Hansen-Love, Paris, Minuit, 1973].

13. Ernst Cassirer, Das mythische Denken, ECW 12, p. 28 [trad. fr. p. 39].

14. Ernst Cassirer, «Die Begriffsform im mythischen Denken », Studien der Warburg Bibliothek 1, LeipzigBerlin, Teubner, 1922. Sur les rapports complexes entre la problématique de Cassirer et l'ordonnancement des volumes dans la bibliothèque Warburg, voir Massimo Ferrari, Ernst Cassirer : Della scuola di Marburgo alla filosofia della cultura, Florence, Olschki, 1996, p. 215-254 ; Salvatore Settis, Warburg continuatus. Descripción de una biblioteca, Barcelone, Ed. La Central-Museo Nacional Centro de Arte Reina Sofía, 2010, p. 28-34. 
leurs dans cette fabuleuse bibliothèque que Cassirer prend connaissance des travaux ethnologiques qui lui permettent de cerner quelque chose comme le fait empirique du mythe, nécessaire à la mise en œuvre de la méthode transcendantale élargie aux premières manifestations de la culture. Il s'agit en l'occurrence de mettre en lumière et de caractériser la « direction de l'objectivation $\$^{15}$ qui s'exprime dans un matériau assez hétérogène ou les grammaires des langues bantoues, mélanésiennes, indiennes ou hamito-sémitiques côtoient les coutumes et rituels des aborigènes d'Australie, de Nouvelle Guinée, des Zuñi d'Amérique du Nord, voire la médecine chinoise et l'astrologie. Si la conscience mythique, telle que Cassirer la conçoit à partir de ce matériau, ne permet pas, comme il l'affirme, de « séparer la sphère de la sensation et de l'intuition objectives de celle du sentiment et de l'affect subjectifs ${ }^{16} \gg$, elle ne fait pas moins preuve d'un mode spécifique de «synthèse du multiple » et de « sériation $\gg(\text { Reibung })^{17}$, autant dire de conceptualisation.

Dans cette étude, Cassirer vise tout particulièrement la formation des concepts relatifs aux catégories d'espace et de cause dans la pensée mythique. Pour illustrer la conception de l'espace qui est à l'œuvre dans les partitions mythiques du monde, il s'appuie sur le «mode de pensée totémique ». Les correspondances qu'il entrevoit à l'intérieur de ce mode de pensée entre l'organisation sociale de la tribu et l'organisation du monde le conduisent à y postuler une corrélation d'essence entre les différentes classes sociales et les domaines de l'espace qui leur correspondent. Là où, aux yeux d'un moderne, il ne serait question que d'analogie qualitative, la pensée mythique pose une communauté d'essence, voire une identité concrète inhérente $^{18}$. Cette essentialisation des rapports entre des objets distincts correspond, selon Cassirer, au primat que la catégorie d'espace exerce sur celle de temps dans la pensée mythique, contrairement à la pensée scientifique qui privilégiera le temps comme forme schématique ${ }^{19}$. La catégorie de cause fait l'objet du même type d'analyse, à la différence près que ce n'est pas le totémisme mais l'astrologie qui fournit le type idéal de la causalité mythique. Dans cette manifestation de la pensée mythique, les deux termes du rapport, « cause » et « effet », sont conçus comme des choses concrètes et reliées entre elles. Là aussi, en privilégiant l'espace comme schème de pensée, les rapports de causalité entre les éléments du divers ne relèvent pas de mécanismes ou de processus dynamiques, mais dépendent des diverses régions de l'espace reliées par la «sympathie universelle ».

On s'aperçoit que dans cette première approche de la forme du mythe, la question est clairement posée en termes de logique transcendantale. Ce qui intéresse surtout Cassirer est la façon dont l'appréhension mythique du monde conditionne les catégories d'espace et de causalité. En même temps, cette étude n'échappe pas à

15. Ernst Cassirer, «Die Begriffsform im mythischen Denken », ECW 16, p. 10 [trad. fr. p. 49].

16. Ibid., p. 17 [trad. fr. p. 55].

17. Ibid., p. 10 [trad. fr. p. 49].

18. Ibid., p. 24-25 [trad. fr. p. 62-63].

19. Ibid., p. 49 [trad. fr. p. 87]. 
l'intentionnalité du système qui vise la connaissance de type scientifique. En effet, une orientation téléologique sous-jacente à l'analyse est indéniable, notamment dans les comparaisons récurrentes entre la forme du concept dans le mythe et sa forme achevée à l'œuvre dans la science moderne de la nature. Ces comparaisons s'appuient sur la distinction, déjà évoquée à propos de la doctrine du concept, entre substance et fonction : alors que le concept mythique est substantiel, le concept scientifique est fonctionnel (relationnel).

En décembre 1924, Cassier parachève le deuxième volume de la PSF, intitulé La pensée mythique, qui sera publié l'année suivante à Berlin. À certains égards, cet ouvrage prolonge la recherche initiée en 1921, si bien que les deux premières parties concernent la façon dont l'appréhension mythique du monde conditionne les catégories d'espace, temps et nombre. Cependant, un élargissement considérable de l'horizon théorique est perceptible dans cet ouvrage : la problématique du concept, qui dans son approche néokantienne était au centre de la première étude, est désormais traitée comme faisant partie d'une question plus vaste et fondamentale touchant au statut propre du «monde » mythique comme objet philosophique et comme « forme structurelle » de l'esprit. La question de la légitimité d'une philosophie de la mythologie, que Cassirer aborde pour la première fois en 1924 dans une conférence dédiée à Paul Natorp ${ }^{20}$, n'est pourtant pas totalement étrangère aux débats du cercle de Marbourg. Ainsi, même si Hermann Cohen n'est pas mentionné dans les pages que Cassirer consacre à cette question, on ne peut pas s'empêcher de voir ici, comme le signale Massimo Ferrari ${ }^{21}$, une certaine prise de position par rapport à Cohen qui, en 1887, avait nié la possibilité que la conscience mythique puisse représenter une direction autonome de la conscience culturelle, contrairement à la conscience scientifique, éthique ou esthétique. Le mythe est, aux yeux de Cohen, la condensation de toutes les puissances de la conscience elle-même, mais non une direction d'objectivation ${ }^{22}$. Pour Cassirer, au contraire, il s'agit d'ériger le mythe en expression symbolique autonome, comme l'avait fait Schelling dans la Philosophie de la mythologie (1856), en séparant de l'étude historique des mythes la réflexion sur l'origine du mythe en tant que tel comme forme de pensée. Pour Schelling, le mythe est un «monde autonome » qui demande à être compris par ses lois immanentes de structure ; son objectivité tient à ce qu'il n'est pas le reflet d'une existence donnée, à ce qu'il ne renvoie à aucun principe, caché ou apparent, extérieur à lui, de quelque nature que ce soit. La vérité philosophique du mythe ne réside donc pas dans son

20. Ernst Cassirer, «Zur Philosophie der Mythologie », in Festschrift für Paul Natorp zum siebzigsten Geburtstage von Scbülern und Freunden gewidmet, Berlin, De Gruyter, 1924. Ce texte deviendra l'introduction à Das mythische Denken.

21. Massimo Ferrari, op. cit., p. 305. Sur le débat du mythe à l'école de Marbourg voir aussi Helmut Holzhey, « Cassirers Kritik des mythischen Bewußtseins », in H. J. Braun, H. Holzhey et W. Orth (éds.), Über Ernst Cassirers Philosopbie der symbolischen Formen, Frankfurt, Suhrkamp, 1988, p. 191-205.

22. Hermann Cohen, Schriften zur Philosophie und Zeitgeschichte, A. Görland et E. Cassirer (éds.), Berlin, Akademie Verlag, 1928, I, p. 397-431. Cohen commente ici l'ouvrage de Friedrich Vischer, Das Symbol (1887). 
contenu, comme le supposaient les théories allégoristes, mais dans son existence même comme totalité intelligible. Dans les termes de Cassirer :

Le phénomène dont il faut rendre compte est en réalité, non pas le contenu de la représentation mythique, mais la signification qu'il a pour la conscience humaine, et la force spirituelle qu'il exerce sur celle-ci. Ce qui fait problème, c'est moins le contenu de la mythologie que l'intensité avec laquelle il est vécu, et la foi qu'on lui accorde au même titre que n'importe quel objet existant effectivement ${ }^{23}$.

Cassirer entend maintenir comme telle l'autonomie objective de l'expression mythique, tout en fondant cette autonomie sur d'autres bases que l'essence de l'absolu. Autrement dit, il s'agit de faire basculer le mythe du domaine de l'idéalisme spéculatif, où Schelling l'avait inscrit, à celui de l'analyse transcendantale. Or ce type d'analyse suppose, comme Cassirer ne cesse de le rappeler, l'existence d'un fait de l'expérience à propos duquel on s'interroge, en régressant à partir de sa réalité, sur ses conditions de possibilité. Mais, sur ce point, le mythe pose un vrai problème méthodologique à la philosophie critique de la culture. D'une part, Cassirer conditionne l'autonomie du mythe à une expérience fondamentale qu'il situe en amont de la création langagière :

C'est une puissance réelle pour la conscience, c'est à dire une puissance qui ne se trouve déjà plus en son pouvoir, qui s'est emparée d'elle dans le mythe. La mythologie naît de quelque chose qui est, au sens strict, indépendant de toute invention, et qui est même, d'un point de vue formel et essentiel, son opposé : un processus nécessaire (pour la conscience), dont l'origine se perd dans une époque au-delà de l'histoire, et auquel la conscience peut, par moments, résister, mais qu'elle ne peut totalement arrêter et encore moins faire régresser ${ }^{24}$.

En excluant d'emblée l'intervention de toute forme de « liberté », de toute instance non causée qui viendrait interrompre le « processus nécessaire », Cassirer situe l'expérience du mythe à un niveau de la conscience dont les expressions échappent au domaine des sciences historiques de la culture. D'autre part, Cassirer refuse de poser la nécessité psychologique du mythe comme le seul contenu réel que l'on puisse objectivement saisir. Il écarte aussi la possibilité d'obtenir le statut propre du mythe par induction à partir des donnés expérimentales de l'ethnologie. À ses yeux, la psychologie et l'ethnologie ont en commun avec la métaphysique le fait de fonder l'unité de la fonction mythique à partir de l'unité d'un substrat préalable. Le statut du mythe comme forme objective de la vie (Lebensform) ne peut être obtenu légitimement ni par la voie d'une science positive, ni par une déduction métaphysique, mais à partir d'une analyse idéelle de sa signification ${ }^{25}$. Or comment saisir cette signification

23. Ernst Cassirer, Das mythische Denken, ECW 12, p. 6 [trad. fr. p. 20].

24. Ibid., p. 7 [trad. fr. p. 20].

25. Ibid., p. 12-16 [trad. fr. p. 26-29]. 
dès lors que le mythe, tel que Cassirer en définit l'objectivité, c'est-à-dire au-delà de la sphère des représentations et de l'histoire, se soustrait de fait à la portée du semiotic turn que l'auteur imprime à la méthode transcendantale ? Nous ne pouvons avoir accès au mythe comme puissance constituante de l'esprit qu'à travers le monde de représentations qu'il produit. Même en fondant l'unité et l'objectivité de la fonction mythique sur le geste, en amont du langage, comme Cassirer l'envisage en donnant la primauté au rituel sur la narration mythique ${ }^{26}$, l'expérience du mythe, qui constitue nécessairement le point de départ de l'analyse, ne se laisse appréhender en dehors du milieu de la représentation et de la mise en sens de l'expérience. Quant à l'orientation idéaliste que Cassirer imprime au système des formes symboliques, qui suppose l'émergence à l'intérieur des chacune de ses parties constituantes d'une instance réflexive propre guidée par l'idée de liberté, elle semble, dans le cas spécifique du mythe, difficilement concevable à l'écart du langage. En effet, le mythe est, dans cette perspective, un des « facteurs déterminants qui permettent à la conscience de se délivrer de la claustration passive dans la sensibilité et de progresser vers la création d'un "monde" organisé selon un principe spirituel qui lui soit propre $\gg^{27 \square}$. Or on peut se demander comment, si ce n'est par le jeu des trois pronoms personnels, le sujet pourrait se délivrer de cette claustration passive. Il semblerait que l'expérience du mythe ne soit compréhensible, du point de vue d'une philosophie critique de la culture, qu'à travers la puissance du langage comme milieu conditionnant l'expressivité. Mais du coup, n'est-ce pas l'autonomie du mythe en tant que forme symbolique qui, dans sa traduction au criticisme, se perd au profit de la priorité systématique du langage ? C'est peut-être la raison pour laquelle Cassirer ne semble pas encore prêt, dans La pensée mythique, à expliciter la position centrale que le langage est amené à occuper dans l'analyse criticiste de la forme mythique.

Ainsi, c'est toujours dans le but de caractériser le mythe comme une production autonome de la pensée, non dérivée de la sphère du langage, que Cassirer contourne la dimension langagière dans l'analyse qu'il fait, dans les deux dernières parties de $\mathrm{La}$ pensée mythique, de la question fondamentale de la découverte de la subjectivité dans la conscience mythique. La méthode de la « phénoménologie de la conscience » qu'il met alors en œuvre se donne pour tâche de mettre en lumière les différents processus au moyen desquels la conscience de soi se construit face au monde physique et social qui l'entoure. Partant une fois de plus du schéma qui oppose le substantialisme de la pensée mythique à l'unité fonctionnelle de la pensée théorique, Cassirer caractérise la

26 Cf. Ernst Cassirer, Das mythische Denken, ECW 12, p. 258 [trad. fr. p. 258]. La thèse de la primauté du rituel sur la narration mythique fut défendue par William Robertson Smith dans ses conférences sur les religions sémitiques (1889), citées ici par Cassirer. Elle fut développée parallèlement par James Frazer dans The Golden Bought (1890), puis trouva un essor considérable dans le domaine grec grâce aux anthropologues dits « ritualistes » de l'école de Cambridge (notamment Jane Harrison, Gilbert Murray et Francis M. Cornford) entre les années 1910 et 1930. Les récits grecs de type étiologique constituaient à leurs yeux la preuve que le mythe est un legomenon qui a toujours pour pendant un drômenon.

27. Ibid., p. 16-17 [trad. fr. p. 30]. 
conscience mythique «par la fluidité et l'inconsistance qui chez elle s'attache encore à l'intuition et à la notion d'existence personnelle $»^{28}$. Le sentiment de soi est confondu dans la conscience mythique avec celui de l'appartenance substantielle à une communauté, laquelle, à son tour, par la corrélation d'essences qu'elle établit avec son espace vital, est fondamentalement liée au monde physique qui l'entoure ${ }^{29}$. Pour décrire le processus idéel de différenciation du moi par rapport à ces instances, Cassirer aurait pu naturellement, dans la lignée de Humboldt, évoquer la fonction pragmatique du langage comme constituante de nos rapports au monde et à nous-mêmes. Mais il semblerait qu'à ce stade de l'analyse, Cassirer évite délibérément de faire appel à la puissance formatrice du langage, comme pour mieux étayer l'autonomie de la forme symbolique du mythe. Deux autres approches, d'inspiration plutôt hégélienne, sont en revanche privilégiées : l'une a trait à la philosophie de la religion, l'autre à la phénoménologie de l'action.

Du point de vue de la philosophie de la religion, telle que Cassirer l'adapte à sa problématique, l'émergence du sentiment d'intériorité individuelle coïncide avec un abandon progressif de la sphère naturelle en direction d'une « intuition d'un royaume spirituel des fins $»^{30}$. Le schéma est celui d'un passage du « moi mythique » (indifférencié du monde physique et de la communauté) au «moi éthique ». On peut se demander, à propos de ce passage du mythos à l'ethos, s'il n'est, en ce qui concerne la systématique des formes symboliques, que l'un des épiphénomènes historiques d'un processus de différenciation qui s'effectue d'abord à l'intérieur de la forme symbolique du langage. L'approche phénoménologique de l'action comme facteur décisif dans l'émergence de la conscience de soi est, quant à elle, plus féconde dans la mesure où Cassirer pose ici les pierres d'une analyse de la conscience prélinguistique qui sera approfondie ultérieurement dans un manuscrit des années 1940 sous la dénomination de Basisphänomene ${ }^{31}$. Dans La pensée mytbique, il est question d'aborder le phénomène de l'action avant même que l'on puisse parler de rationalité instrumentale. Comme l'affirme Cassirer :

Beaucoup plus que la contemplation théorique, c'est l'action (das Tun) qui constitue le centre à partir duquel va se déployer pour l'homme l'organisation spirituelle de la réalité. C'est par elle seulement que les domaines de la subjectivité et de l'objectivité [...] commenceront à s'écarter l'un de l'autre. La rupture entre ces deux mondes et la frontière entre le « moi » et le «non-moi » apparaissent de plus en plus clairement à mesure que progresse la conscience de l'action ${ }^{32}$.

28. Ibid., p. 186 [trad. fr. p. 189].

29. Il n'est pas inutile de signaler en passant que cette équation sera à nouveau mobilisée par Cassirer dans son dernier livre pour caractériser le nazisme comme résurgence d'une forme de pensée mythique. Voir The Myth of the State (1946), ECW 25 [trad. fr. de B. Vergely, Le mythe de l'état, Paris, Gallimard, 1993].

30. Ernst Cassirer, Das mythische Denken, ECW 12, p. 199 [trad. fr. p. 201].

31. Manuscrit édité par John Michael Krois et Oswald Schwemmer dans les Nachgelassene Manuskripte und Texte, ECN 1, p. 111-195.

32. Ernst Cassirer, Das mythische Denken, ECW 12, p. 183 [trad. fr. p. 187]. 
Les phénomènes de base, à savoir le « je »(Ich-Phänomen), l'« activité » (Wirkens-Phänomen) et l'« œuvre » (Werk-Phänomen), dont deux d'entre eux s'insinuent déjà en 1924 dans La pensée mythique, seront théorisés ultérieurement comme phénomènes «précédant toute pensée ou inférence ${ }^{33}$ ». Cassirer précise dans le manuscrit mentionné, que « ce sont eux-mêmes qui s'ouvrent (aufschliessen), c'est-àdire ceux qui révèlent (offenbaren) et rendent manifeste (manifest-machen). Ils sont les "originär-gebenden" Intentionen dans le sens de Husserl ${ }^{34} \gg$. Ce n'est pas le lieu ici de commenter le tournant théorique, perceptible dans ce manuscrit, qui vers la fin de sa vie semblait conduire Cassirer à une réorganisation du système des formes symboliques en direction de problèmes phénoménologiques (au sens de Husserl) et psychologiques (au sens de Natorp) ${ }^{35}$, mais il n'est pas inutile de noter que les phénomènes de base que sont le «je » et l'« action » font leur première apparition dans son œuvre comme appartenant en propre à la sphère symbolique du mythe. Si l'on tient compte du fait que la première moitié de l'ouvrage La pensée mythique s'inscrit pleinement dans une problématique transcendantale (conditionnement des catégories par la pensée mythique), le changement d'orientation qui intervient dans la deuxième moitié du livre, consacrée à des phénomènes censés précéder la mise en forme de la pensée par le langage, permet de mesurer l'embarras méthodologique que suscite le mythe comme forme symbolique. Il semblerait que plus on cherche à cerner l'autonomie de la forme mythique par rapport à celle du langage, plus il devient difficile de reconstruire l'expérience objective du mythe nécessaire au déploiement de la méthode transcendantale.

Les difficultés méthodologiques sous-jacentes aux approches mises en œuvre dans La pensée mythique ne peuvent être surmontées sans l'élucidation préalable du rapport constitutif qu'entretiennent le mythe et le langage. Ce n'est donc pas par hasard si pendant qu'il parachève le deuxième volume de la PFS Cassirer travaille simultanément à Langage et mythe. Cette étude, qui se veut en partie un commentaire de l'ouvrage Götternamen de Hermann Usener, porte, comme son titre l'indique, sur la corrélation problématique entre ces deux formes symboliques. Comme on l'a déjà remarqué, l'hypothèse que l'élément mythique et l'élément linguistique se rapportent corrélativement l'un à l'autre et soient même indissolublement unis soulève, dans la perspective d'une théorie générale de la culture, le problème majeur de leur différence spécifique. Considérant la disposition thématique de la PFS en trois volumes relativement autonomes, consacrés respectivement au langage, au mythe et à la phénoménologie de la connaissance, on comprend que la question du rapport mythe/langage puisse faire l'objet d'un livre à part, comme un appendice qui viendrait élucider le lien systématique entre les thèmes des deux premiers volumes de l'œuvre. Sa forme, à mi-chemin entre une longue recension critique et une digression

33. Ernst Cassirer, Nachgelassene Manuskripte und Texte, ECN 1, p. 132.

34. Ibid.

35. Sur les Basisphänomene et leur relation avec la théorie de la symbolisation, voir Sebastian Ullrich, Symbolischer Idealismus. Selbstverständnis und Geltungsanspruch von Ernst Cassirers Metaphysik des Symbolischen, Hambourg, Felix Meiner Verlag, 2010, p. 75-86. 
théorique, a sans doute conduit certains spécialistes à sous-estimer l'importance de ce petit volume au sein de l'œuvre de Cassirer ${ }^{36}$. Publié en 1925 dans la série Studien der Bibliothek. Warburg, quelques mois après la parution du deuxième volume de la PFS, Langage et mythe se saisit pourtant de la question fondamentale laissée en suspens à la fin de La pensée mythique:

L'interrogation [de la philosophie des formes symboliques] concerne moins la communauté d'origine que l'identité de structure. Elle ne cherche pas entre le langage et la religion l'unité cachée d'un fondement et doit au contraire se demander si entre eux, entre ces deux productions parfaitement autonomes et uniques en leur genre, on ne peut découvrir une unité de fonction. Si cette unité existe, il convient de ne la chercher nulle part ailleurs que dans une direction fondamentale de l'expression symbolique elle-même, dans une règle interne qui en détermine le développement et le déploiement ${ }^{37}$.

Toute la pertinence méthodologique de la philologie spéculative d'Usener dans la problématique de Cassirer est condensée de façon résolument transcendantale dans ces quelques lignes. Nous verrons par la suite que l'apport effectif du philologue consiste précisément à situer cette « règle interne déterminant le développement et le déploiement » des mondes symboliques dans le langage.

\section{Le recours à la philologie}

La problématique de la corrélation entre langage et mythe, dont on a pu mesurer la difficulté théorique qu'elle pose à la philosophie des formes symboliques, a de son côté une riche histoire qui est indissociable de la constitution de la philologie comme science historique des textes, différenciée de la philosophie

36. Dans les études consacrées à la forme du mythe chez Cassirer que j'ai pu consulter, la position qu'occupe Langage et mythe dans son œuvre ne fait l'objet d'aucun commentaire, tout comme le rôle joué par la philologie d'Usener dans sa conception du rapport mythe/langage. Cf. Helmut Holzhey, op. cit. ; Dominic Kaegi, «Ernst Cassirer über Mythos und symbolische Form », in E. Rudolph (éd.), Mythos zwischen Philosophie und Theologie, Darmstadt, Wiss. Buchges., 1994, p. 167-199; Thomas Vogl, Die Geburt der Humanität. Zur Kulturbedeutung der Religion bei Ernst Cassirer, Hambourg, Felix Meiner Verlag, 1999, p. 92-149 ; Cornelia Richter, « Symbol-Mythos-Religion. Zur Funktion der Religion in der Philosophie Ernst Cassirers », in D. Korsch et E. Rudolph (éds.), Die Prägnanz der Religion in der Kultur, Tübingen, Mohr Siebeck, 2000, p. 5-32. Seule exception, l'étude d'A. Poma (« Il mito nella filosofia delle forme simboliche di Ernst Cassirer », Filosofia 31, 1980, p. 672), qui affirme cependant que Langage et mythe « costituisce la fase dell'indagine cassireriana sul mito immediatamente precedente ai risultati piú compiuti e complessi che appaiono nella Philosophie der symbolischen Formen. Tuttavia, non bisogna supravvalutare l'importanza di quest'opera, perche si corre altrimenti il rischio di travisare, almeno parzialmente, le conclusioni a cui giunge la filosfia di Cassirer, soprattutto in merito al rapporto tra mito e linguagio ».

37. Ernst Cassirer, Das mythische Denken, ECW 12, p. 298 [trad. fr. p. 297]. 
et de la théologie ${ }^{38}$. Dans ce débat, la priorité effective que l'on donnait à l'une des formes, mythe ou langage, sur l'autre, déterminait en grande mesure la manière dont chaque philologue définissait la rationalité du sens d'une expression langagière. Je n'ai pas l'intention ici de revisiter les fondements épistémologiques de la philologie ; plus modestement, je voudrais évoquer deux points de vue paradigmatiques, l'un, celui de Friedrich Creuzer, donnant la priorité au symbole mythicoreligieux, l'autre, celui de Gottfried Hermann, donnant la priorité au langage. La mise en lumière de leurs arrière-plans théoriques devrait nous permettre de mieux mesurer l'originalité de la position d'Usener ainsi que son apport à la problématique spécifique de Cassirer.

La Symbolique $e^{39}$ de Creuzer constitue certainement l'un des textes les plus influents dans le mouvement de revalorisation du mythe comme forme de pensée à l'époque romantique. Schelling, dont on a déjà mentionné l'influence sur la position théorique de Cassirer, s'appuiera sur le matériau réuni par Creuzer pour ériger le mythe en «monde autonome » de l'esprit. Pour expliquer le fait religieux, Creuzer pose comme état originel de la conscience un rapport substantiel au divin et donc une dépendance absolue vis-à-vis d'une nature indifférenciée et transcendante. Cette expression religieuse, qui prend la forme d'un monothéisme, conduit Creuzer à supposer l'existence d'une théologie originelle, qu'il situe géographiquement en Orient. Là, des prêtres auraient prêché la théologie première au moyen de symboles qui s'articulaient dans des énoncés brefs capables d'impressionner la foule. Conquérant progressivement leur autonomie par rapport à la révélation, ces énoncés se sont développés en mythes puis en poèmes perdant dans ces transformations leur sens profond. La démarche de Creuzer consiste en la définition d'une origine sémantique unique dont il reconstruit le processus de perte et de différenciation historique. De son point de vue, non seulement le symbole religieux échappe à toute conceptualisation, mais, en tant que rapport authentique à la réalité, il précède toute forme d'élaboration langagière. «Dans le symbole, dit-il, notre âme se sent saisie et la nécessité de la nature la domine ${ }^{40}$. Cette caractérisation de l'expression religieuse et de l'inévitable processus de sa dégénérescence dans un savoir allégorique font partie d'un schéma néoplatonicien que Cassirer ne cesse de dénoncer chez d'autres auteurs, en particulier chez Max Müller ${ }^{41}$.

38. C'est en effet une querelle sur l'origine des mythes, opposant Friedrich Creuzer (1771-1858) à Gottfried Hermann (1772-1848), dans les premières décennies du XIX ${ }^{\mathrm{e}}$ siècle, qui passe pour l'acte de naissance de la philologie critique. Pour une analyse des enjeux de la dispute, voir Pierre Judet de La Combe, « La querelle philologique du mythe. Les termes d'un débat en Allemagne et en France au début du siècle dernier », Revue germanique internationale 4, 1995, p. 55-67.

39. Friedrich Creuzer, Symbolik und Mythologie der alten Völker, besonders der Griechen, LeipzigDarmstadt, Leske, 1810-1812.

40. Ibid., p. 71.

41. Max Müller utilise l'analyse linguistique et étymologique comme un moyen pour éclairer la nature de certaines figures mythiques, celles en particulier qui appartiennent à la religion védique. Pour Müller, le mythe est en rapport avec un manque fondamental du langage. Toute désignation linguistique 
C'est contre cette conception «mystique » de la mythologie que s'insurge Gottfried Hermann, dont le point de vue définit en quelque sorte les tâches de la philologie critique. Hermann va tout d'abord postuler une adéquation entre les catégories de l'entendement et les catégories linguistiques. De fait, les termes utilisés dans sa grammaire, dans sa métrique et dans ses écrits critiques sont dérivés de l'analytique transcendantale. Pour lui, les mythes ne renvoient à aucune théologie cachée et le caractère étrange des créatures mythiques n'exprime pas le sentiment de dépendance vis-à-vis d'une nature insaisissable, comme c'était le cas pour Creuzer ; bien au contraire, le mythe se laisse réduire à des concepts clairs pour peu qu'on reconstitue l'étymologie des noms des dieux. Sous ces noms se cacheraient en effet des propositions grammaticales univoques qui sont les prédicats multiples de substances identifiables dans les cosmologies mythiques ${ }^{42}$. Pour Hermann, il ne peut y avoir de priorité symbolique en deçà de la langue, on est donc fondé à lire les histoires primitives des dieux (c'est en fait d'Homère et d'Hésiode qu'il s'agit ici) comme l'expression d'un premier rapport langagier aux phénomènes naturels. Cette position philologique, kantienne à sa manière, rend impossible toute approche du mythe comme forme originaire et autonome de la pensée.

Ces deux thèses, que j'ai choisies parmi d'autres exemples possibles à cause du tranchant de leur opposition, illustrent bien le dilemme auquel on est confronté lorsqu'on cherche à définir l'origine de l'expression symbolique en termes de priorité temporelle et substantielle. Or un véritable changement de perspective dans le traitement de la corrélation mythe/langage a lieu en 1896 avec la publication de Götternamen, Versuch einer Lebre von der religiösen Begriffsbildung par Hermann Usener ${ }^{43}$. Ici, le

est nécessairement équivoque et c'est dans cette équivocité, dans cette « paronymie » des mots qu'il faut chercher la source et l'origine de tous les mythes. Un schéma de néoplatonicien sous-tend la thèse de Müller : «La mythologie est inévitable, elle est une nécessité inhérente au langage, si nous reconnaissons dans le langage la forme extérieure de la pensée : elle est, en un mot, l'ombre obscure que le langage jette sur la pensée et qui ne disparaîtra jamais, aussi longtemps que langage et pensée ne coïncideront pas entièrement - ce qui ne pourra jamais être le cas. [...] La mythologie, au sens le plus pur du terme, est le pouvoir que le langage exerce sur la pensée, dans toutes les sphères possibles de l'activité spirituelle ». Cf. Über die Philosophie der Mythologie, 1876, cité par Cassirer, Das mythische Denken, ECW 12, p. 27 [trad. fr. p. 39].

42. Cf. Gottfried Hermann et Friedrich Creuzer, Briefe über Homer und Hesiodus, Heidelberg, 1818. Voir à ce sujet, Pierre Judet de La Combe, op. cit. ; Antonio La Penna, « Sugli inizi della filologia classica "positivistica” in Germania », in Antonio Santucci (éd.), Scienza e filosofia nella cultura positivistica, Milan, Feltrinelli, 1982, p. 427-445.

43. Quand Usener aborde la question des noms des dieux, il a déjà exploré tout au long de sa carrière les diverses potentialités de la philologie comme science historique des textes. On peut citer des contributions remarquables dans une grande variété de domaines : dans l'édition critique, on notera son Épicure (1887) ; sa thèse, Analecta Theophrastea (1858) dirigée par Brandis, fournit le modèle des Doxographi Graeci à son disciple Diels ; dans l'interprétation de figures mythiques romaines, on citera «Italische Mythen » (1875) ; dans le champ l'anthropologie religieuses, ses travaux sur la légende hagiographique de Sancta Pelagia (1877) et sur l'origine des fêtes de la Nativité (1889), posent déjà la question de la survivance des mythes antiques qui sera déterminante pour son disciple Warburg. Pour un aperçu de l'ensemble de l'œuvre d'Usener, voir : H. J. Mette et A. Thierfelder (éds.), Lustrum 22, 19791980 ; Arnaldo Momigliano, Studies on Modern Scholarship, trad. de T. J. Cornell, Berkeley, University 
mythe relève d'un type spécifique de représentation qui s'inscrit dans la problématique large d'une théorie historique de la connaissance : « Notre théorie de la connaissance sera privée de son fondement nécessaire jusqu'à ce que la linguistique et la mythologie aient éclairci le processus de la représentation spontanée et inconsciente ${ }^{44}$. » À cet égard, la philologie d'Usener s'inscrit résolument du côté des sciences de l'esprit dans le partage épistémologique proposé par Dilthey, où elle destinée à jouer un rôle fondamental dans la comprébension des objets historiques : selon Usener, on peut effectivement affirmer, en paraphrasant Kant et le mathématicien Leo Königsberger, que si les sciences de la nature sont des sciences dans la mesure où elles contiennent des mathématiques, à plus forte raison, les disciplines historiques sont historiques dans la mesure où elles contiennent de la philologie ${ }^{45}$. Cependant, le terme « philologie » employé ici par Usener demande à être précisé, car il ne renvoie pas au sens traditionnel du terme, c'est-à-dire à la science qui a pour objet l'historicité individuelle des textes, où l'on affirme le primat de la volonté expressive de l'auteur sur la langue. La philologie dont il est ici question privilégie plutôt l'analyse de la puissance anonyme de la langue comme milieu conditionnant de l'expressivité. Comme Usener le dit lui-même dans une lettre à son ancien disciple Wilamowitz, qui était l'incarnation même de la philologie critique, « vous cherchez la création de la volonté dans l'histoire, moi, le devenir involontaire, inconscient ${ }^{46} \gg$. Si l'on devait caractériser ces deux positions philologiques dans les termes de la célèbre distinction introduite par Schleiermacher dans l'Herméneutique générale (1809-1810), on dira qu'Usener privilégie dans Götternamen l'interprétation « grammaticale » au sens où c'est la langue qui prédomine sur l'individu, tandis que dans la forme de compréhension philologique qui va s'imposer dans la seconde moitié du XIX ${ }^{e}$ siècle, de Hermann à Wilamowitz, appelée « technique » ou « psychologique », c'est la volonté expressive de l'auteur qui constitue le véritable objet d'étude. Chez Usener, l'accent est mis sur la puissance formatrice du langage et c'est en ce sens qu'il définit la mythologie comme une « morphologie des représentations religieuses ${ }^{47} »$.

\section{La relocalisation du langage dans le système}

La méthode philologique d'Usener soulève la question de la relation que l'on doit établir entre langage et histoire, dont on sait l'importance qu'elle revêt pour la

of California Press, 1994, p. 251-266 ; G. Arrighetti (et alii), Aspetti di Hermann Usener filologo della religione, Pise, Giardini, 1982. Sur la postérité d'Usener, voir Antje Wessels, Ursprungszauber. Zur Rezeption von Hermann Usenrs Lebre von religiösen Begriffsbildung, Berlin-New York, De Gruyter, 2003.

44. Hermann Usener, Götternamen, Bonn, F. Cohen, 1896, p. 321.

45. Hermann Usener, « Philologie und Geschichtwissenschaft » (1882), in Vorträge und Aufsätze, Leipzig-Berlin, Teubner, 1914, (p. 1-35) p. 29 ss.

46. Lettre d'Usener à Wilamowitz, datée le 20/7/1887, in Usener und Wilamowitz. Ein Briefwechsel 1870-1905, Leipzig, 1994, n. 5, p. 7.

47. Hermann Usener, Götternamen, p. VI. Voir aussi Mythologie (1904) in Vorträge und Aufsätze, (p. 37-65) p. 57. 
philosophie des formes symboliques de Cassirer. Si, dès la préface de Götternamen, Usener prend explicitement ses distances avec la philosophie spéculative, sa méthode ne correspond pas pour autant à une historiographie positive. En effet, une philologie qui se donne pour tâche de restituer « les processus spirituels des hommes préhistoriques $^{48} \gg$ dans l'horizon d'une théorie de la connaissance peut difficilement se contenter de données empiriques. Un aspect fondamental de l'ouvrage d'Usener à propos duquel Cassirer ne cache pas son enthousiasme est justement le rapport que l'auteur établie entre historicité et systématicitét9. La reconstruction des premières représentations religieuses de l'humanité dépend chez Usener d'une loi générale censée régir le développement historique de l'articulation linguistique du monde. Cette loi postule la coexistence de deux forces aux tendances opposées, l'une va dans le sens de la particularisation, c'est celle qui caractérise le mieux la conscience mythique, l'autre va vers la généralisation, c'est celle qui caractérise la pensée conceptuelle. L'interaction de ces deux tendances immanentes au langage, dont la parenté avec les principes d'homogénéité et de spécification chez Kant est manifeste, peut être saisie dès les débuts de l'histoire de la pensée pour peu qu'on y inclut le moment du mythe :

Ce pas des perceptions singulières au concept générique est bien plus grand que ne nous permettent de le supposer notre formation scolaire et une langue qui pense pour nous. Il est si grand que je suis incapable de concevoir quand et comment les hommes auraient pu le franchir, si le langage, à l'insu de l'homme, n'avait préparé et provoqué ce processus. C'est le langage qui, de la multitude d'expressions particulières équivalentes, en laisse progressivement sortir une qui étend son domaine sur un nombre croissant de cas, jusqu'à ce qu'elle soit apte à les embrasser tous et à devenir un concept universel ${ }^{50}$.

La puissance formatrice du langage, responsable du passage des concepts particuliers (Sonderbegriffe) aux concepts universels (Gattungsbegriffe), trouve chez Usener un corrélat historique dans l'évolution des représentations des dieux. L'intérêt que la reconstruction de cette évolution présente pour Cassirer consiste essentiellement en ce qu'elle n'est pas conçue par Usener de manière linéaire, mais selon un rapport dialectique entre les deux tendances opposées du langage qui œuvrent à l'intérieur de la forme mythique. L'hypothèse que Cassirer élabore à partir de sa lecture d'Usener est que mythe et langage émergent simultanément d'un même acte fondamental de l'esprit de concentration et d'amplification d'une perception sensorielle. Or, si tous les deux partent de la même impulsion de mise en forme symbolique, ils tendent vers des directions opposées dans le cours de leur différenciation en un monde d'images, d'une part, et un monde linguistique, de l'autre ${ }^{51}$. Les

\footnotetext{
48. Ibid., p. VII.

49. Ernst Cassirer, Sprache und Mythos, ECW 16, p. 239 ss. [trad. fr. p. 24 ss.].

50. Hermann Usener, Götternamen, p. 321 s.

51. Ernst Cassirer, Sprache und Mythos, ECW 16, p. 304-306 [trad. fr. p. 112-114].
} 
images mythiques se caractérisent par la condensation d'impressions individuelles dont le sens est essentiellement lié à leur contexte d'origine, tandis que la direction symbolique du langage se caractérise par la généralisation des cas individuels et l'élaboration d'un monde articulé en catégories. Mais comme le montre la typologie des dieux élaborée par Usener, ces deux directions symboliques opposées sont simultanément à l'œuvre dans chacune des étapes de la formation des représentations mythico-religieuses.

Pour Usener, la période de la pensée mythique la plus reculée est caractérisée par la formation des « dieux de l'instant » (Augenblicksgötter). Ils ne personnifient pas une puissance générale de la nature, aucun trait ni état uniformément répété ne s'y rattache. C'est au contraire une excitation instantanée chez le sujet, un contenu de l'âme qui disparaît aussi rapidement qu'il apparaît et qui, en s'objectivant et en se déchargeant vers l'extérieur, crée la figure du dieu de l'instant. La tension entre le signe linguistique et le contenu intuitif auquel il se rapporte est ici pratiquement absente. Quand, par l'effet d'une impression forte, on attribue à un objet ou à l'état dans lequel on se trouve la valeur du divin, alors le dieu de l'instant est ressenti et créé ${ }^{2}$. C'est ici que s'amorce la distinction entre le sacré et le profane, que Cassirer considère comme l'une des oppositions fondamentales de la pensée mythique ${ }^{53}$. Mais une forme de négation des dieux de l'instant survient avec la deuxième série de divinités, les « dieux spéciaux » (Sondergötter), qui n'a pas son origine dans la sensation momentanée, mais dans l'activité durable et ordonnée de l'homme. Celui-ci cesse d'être à la merci d'impressions extérieures quand, par sa propre volonté, il participe au cours des événements pour le régler sur son désir et son besoin. Cette régularisation porte en elle-même sa mesure et sa périodicité : elle consiste dans la répétition uniforme, à certains intervalles de temps, de la même série d'activités à laquelle s'attache un seul et même effet durable, niant par cette étendue régulière l'emprise de l'instant. Comme auparavant avec les dieux de l'instant, le moi ne peut prendre conscience de cet agir qu'en le projetant vers l'extérieur et en le posant devant soi dans une formation langagière. Usener établie ici une forme d'homologie entre l'agir et le discourir : les dieux spéciaux restent liés à un domaine spécifique bien déterminé et n'ont pas de fonction générale, mais à l'intérieur de la sphère d'activité qui leur est assignée, ils ont déjà un caractère et une durée stables. Là où le dieu spécial est appréhendé d'abord, il possède un certain nom, procédant de la sphère particulière d'activités à laquelle il préside. Aussi longtemps que ce nom est encore compris dans sa signification d'origine, la limitation du nom correspond à celle du dieu. Mais il en va autrement lorsque la dénomination du dieu perd son rapport avec le vocabulaire vivant. Alors le nom n'évoque plus la représentation singulière, mais devient un nom propre, l'idée d'une personnalité déterminée. Apparaissent alors les « dieux personnels » (Personalgötter), qui peuvent agir et pâtir comme les hommes, qui sont actifs de différentes manières et ne se réduisent pas à une seule action. Pour Usener, ils ont

52. Hermann Usener, Goternämen, p. 279-301.

53. Ernst Cassirer, Das mythische Denken, ECW 12, p. 112-116 [trad. fr. p. 122-126]. 
acquis la généralité et l'autonomie référentielle des symboles nominatifs ${ }^{54}$. Cassirer poursuit les développements de cette loi de formation des concepts mythico-religieux jusqu'à la déduction linguistique du monothéisme, qui marquerait le moment où la pensée parvient à un point où la multiplicité des attributs divins et des noms des dieux « ne lui suffisent plus, et où l'unité du mot lui devient un moyen grâce auquel elle cherche à parvenir à l'unité du concept de dieu ${ }^{55} \gg$.

Plutôt que de séparer dans la pensée mythico-religieuse le moment de l'image de celui du concept, la dynamique issue de l'analyse d'Usener les associe dialectiquement. L'émancipation progressive du sensible qui est à l'œuvre ici n'implique pas quelque chose comme un abandon de l'image et un passage définitif au pur concept, selon le schéma classique «du mythos au logos » : non seulement le concept est issu d'une image, mais dans «l'exhaustivité virtuelle de ses déterminations ${ }^{56}$ » il n'est, sur un autre plan, qu'une nouvelle image, elle-même appelée à devenir concept. Sur ce point, il n'est pas inintéressant de remarquer que Cassirer, comme l'a montré André Laks, déploie un schéma dialectique analogue dans l'étude qu'il consacre en 1925 la même année que Langage et mythe - aux débuts de la philosophie grecque ${ }^{57}$. Pour Cassirer, ce motif dialectique peut être avancé comme hypothèse pour comprendre non seulement l'évolution des contenus du mythe, mais aussi celui de sa forme symbolique en tant que telle :

Les différents moments de l'évolution du mythe, loin de se succéder purement et simplement, s'opposent, et souvent de manière assez vive. Le progrès du mythe ne consiste pas à développer et à compléter certains caractères, certaines déterminations des stades antérieurs, mais à les nier. Et même à les anéantir purement et simplement. Et cette dialectique n'intervient pas seulement pour transformer les contenus de la conscience mythique : elle en règle aussi la « forme interne ». Elle s'en empare même, pour la métamorphoser de l'intérieur, de la fonction mythique en tant que telle, du processus de construction des mythes ${ }^{58}$.

Cette hypothèse sur l'évolution d'une forme symbolique donnée n'est envisageable que si l'on assigne au langage, à l'instar d'Usener, une position rectrice dans le système ; une place qui lui revient en vertu du fait que c'est en lui que s'élaborent,

54. Hermann Usener, Götternamen, p. 323. Cassirer ne manque pas sur ce point de relever un paradoxe dans la corrélation qu'Usener tente d'établir entre le mythe et le langage lorsque ce dernier met en parallèle la métamorphose des dieux spéciaux en dieux personnels, d'un côté, et la tendance du langage à passer des représentations singulières aux générales, de l'autre. Voir Das mythische Denken, ECW 12, p. 241-242 [trad. fr. p. 241-242].

55. Ernst Cassirer, Sprache und Mythos, ECW 16, p. 290 [trad. fr. p. 93].

56. J'emprunte cette phrase à André Laks, Introduction à la «philosophie présocratique », Paris, PUF, 2006, p. 149.

57. Ibid., p. 134-149. Cf. Ernst Cassirer, « Die Philosophie der Griechen von den Anfängen bis Platon $\gg(1925)=$ ECW 16, p. 313-467.

58. Ernst Cassirer, Das mythische Denken, ECW 12, p. 275 [trad. fr. p. 275]. 
par le jeu des attributs et des négations, les déterminations contrastées des représentations et des images du monde.

La position privilégiée que le langage acquiert par rapport aux autres formes symboliques traduit, comme nous l'avons déjà remarqué, une inflexion par rapport à la perspective néokantienne d'une théorie de la culture où les formes symboliques sont toutes considérées comme étant également originaires et incommensurables. Du point de vue de la cohérence et de la visée du projet de Cassirer, dont on a évoqué au début de cet article la difficulté principielle qui le traverse, on peut se demander si la relocalisation du langage ne trahit pas l'évolution d'une théorie générale de la culture en une phénoménologie de la culture. En ce sens, le motif dialectique donnerait la clé logique du déploiement unifié de ce qui fonde la vie humaine dans ses réalisations historiques successives. Certes, avant d'être usenerien, ce schéma est hégélien, en revanche, ce que l'on peut identifier comme l'apport indéniable de la philologie spéculative, c'est la reconnaissance de l'immanence de cette dialectique au langage. Désormais, la tâche générale que Cassirer assigne à la philosophie est de « comprendre [les formes symboliques] dans leur interpénétration (Ineinander) et à les concevoir dans leur relative dépendance (Abhängigkeit) comme dans leur relative autonomie (Selbständigkeit) ${ }^{59} \gg$. On constate dans cette formule programmatique tirée de Langage et mythe que la tendance n'est plus à souligner l'objectivité propre et non dérivée de chacune des parties du système, mais à leur accorder une relative autonomie qui suppose implicitement une relative dépendance par rapport au langage. La position paradigmatique que celui-ci occupe dans le système permet d'envisager l'extension du motif dialectique à l'ensemble des relations entre les diverses activités symboliques et de rendre compte de la dynamique de leur succession historique :

Si nous nous sommes efforcés jusqu'à présent de découvrir la racine commune aux formations conceptuelles du mythe et du langage, il s'agit maintenant de nous demander comment ce lien se manifeste dans la structure du « monde » mythique et dans celle du « monde » linguistique. Il s'y révèle une loi qui vaut d'une même façon pour toutes les formes symboliques et qui détermine leur développement de façon essentielle. Dans leur ensemble, elles n'apparaissent pas d'emblée comme des configurations séparées, existant par soi et identifiables, mais se détachent très progressivement de cette terre commune, le mythe. Tous les contenus de l'esprit, quand bien même nous devons leur attribuer un domaine propre d'un point de vue systématique et leur donner pour fondement un propre « principe » autonome, ne nous sont donnés, à titre de faits, que dans cette interpénétration. [...] Là où les formes singulières cherchent à sortir du tout et à s'opposer les unes aux autres dans la prétention à un caractère spécifique, elles semblent se déraciner elles-mêmes et abandonner une partie de leur propre essence. Progressivement nous apprenons que cet abandon est un moment nécessaire de leur autodéploiement, que la négation renferme le germe d'une nouvelle position, que la séparation

59. Ibid., p. 234 [trad. fr. p. 17]. 
devient elle-même le fondement d'une nouvelle réunion qui nait de présupposés d'une autre espèce ${ }^{60}$.

On entrevoit dans ce passage le sens que l'expression «phénoménologie de la connaissance » prendra dans le titre du troisième volume de la PFS. Corroborant le tournant théorique engagé dans Langage et mythe, il s'agira pour Cassirer d'embrasser la totalité des formes de l'esprit contenues potentiellement dans la forme du mythe. À ce propos, Cassirer revendique la parenté de son projet avec celui de Hegel qui, dans la préface à la Phénoménologie de l'esprit, affirmait que la totalité n'est perceptible que dans le passage d'une forme de l'esprit à une autre, car la vérité du « tout » ne peut s'offrir d'un seul coup, mais se déploie selon un mouvement qui lui est propre ${ }^{61}$. Sur ce modèle hégélien, il convient cependant de relever deux distinctions importantes pour notre propos. D'une part, à la différence de Hegel, le principe dynamique qui chez Cassirer préside à la différenciation des mondes symboliques n'est pas d'ordre métaphysique, mais de nature langagière. De l'autre, la pensée linguistique de Cassirer se distingue de celle d'Usener sur un point décisif : alors que ce dernier, comme nous l'avons déjà signalé, privilégie une forme d'évolution des représentations où la langue, dans un processus croisant d'abstraction, construit les concepts à l'insu des individus, Cassirer affirme, dans la lignée de Humboldt, que le langage « provient de la subjectivité et même de l'individualité des locuteurs ${ }^{62}$, évitant par là la disparition du sujet dans une histoire destinale des formes symboliques. Tant que cette conception humboldtienne du langage est préservée, le sont aussi l'identité et l'autonomie du sujet transcendantal. Une telle phénoménologie de la culture ne demande qu'à être mise à l'épreuve, non pas quant à sa légitimité abstraite, que j'ai voulu ici reconstruire à partir de la lecture que Cassirer fait d'Usener, mais par des analyses proprement historiques et philologiques susceptibles de donner des nouveaux contenus concrets à l'idée de différenciation.

60. Ibid., p. 266 [trad. fr. p. 61].

61. Ernst Cassirer, Phänomenologie der Erkenntnis = ECW 13 p. VIII-IX [trad. fr. 8-9].

62. Ernst Cassirer, « Die kantischen Elemente in Wilhelm von Humboldt Sprachphilosophie » (1923), ECW 16 (p. 105-133), p. 122. Ce point est pertinemment développé par Marc de Launay dans « Histoire et dialectique d'une forme symbolique », texte qu'il publie dans ce même recueil. 